\title{
How Environmental Agents Influence the Aging Process
}

\author{
Meryl H. KaroL* \\ Department of Environmental and Occupational Health, University of Pittsburgh, Pittsburgh, PA, USA
}

(Received April 15, 2009; Revised April 21, 2009; Accepted April 22, 2009)

\begin{abstract}
Aging is a multifaceted biological process that affects all organs and organ systems of the body. This review provides an up-to-date analysis of this highly exciting, rapidly changing field of science. The aging process is largely under genetic control but is highly responsive to diverse environmental influences. The genes that control aging are those that are involved with cell maintenance, cell damage and repair. The environmental factors that accelerate aging are those that influence either damage of cellular macromolecules, or interfere with their repair. Prominent among these are chronic inflammation, chronic infection, some metallic chemicals, ultraviolet light, and others that heighten oxidative stress. Other environment factors slow the aging process. Included among these agents are resveratrol and vitamin D. In addition, dietary restriction and exercise have been found to extend human lifespan. The various mechanisms whereby all these agents exert their influence on aging include epigenetic modification, chromatin maintenance, protection of telomeres, and anti-oxidant defense, among others. The complex process of aging remains under continued, intense investigation.
\end{abstract}

Keywords: Aging, Environment, Oxidative stress, Inflammation, Heavy metals, Resveratrol, Calorie restriction

\section{INTRODUCTION}

Aging is a complex biological process that represents cumulative structural and functional changes in an organism over time. It is a genetically-regulated process that is responsive to environmental influences. Agents present in indoor and outdoor environments and in the diet affect the aging process as does exercise, past illnesses, and more. Age-related changes occur in all tissues of the body and affect the functioning of all body systems. The biological mechanisms underlying aging are still unclear. As indicated in Table I, the last half-century has witnessed major advances in understanding the process and components of aging. We now recognize the aging process to be the gradual accumulation of molecular and cellular damage.

With very few exceptions, all multicellular animals age. ${ }^{1}$ Our understanding of aging has benefited enormously from studies using a variety of simple animal species. One

${ }^{*}$ Corresponding author

Tel: +1-412-431-6688

E-mail: mhk@pitt.edu observation is that after an organism has attained its maximal reproductive competence, its biological functions begin to deteriorate (Farooqui and Farooqui, 2008). Cognitive changes relevant to aging usually begin in mid-life and continue with advancing age. The brain shrinks with age. Neuronal axons that connect different regions of the brain deteriorate with time, weakening information processing. Memory fades.

Several theories have been proposed to explain the aging process. As indicated in Table II, the Free Radical Theory proposes that aging results from accumulated macromolecular damage. Mitochondrial reactive oxygen species are considered a major cause of damage with its most vulnerable targets being DNA, other template molecules and extracellular matrix proteins. (Pamplona, 2008; Campisi and Vijg, 2009). Accumulated damaged molecules impair cell function. Animal models in which macromolecular damage accumulates at an accelerated rate have shown premature aging phenotypes and a shortened life span (Campisi and Vijg, 2009). Uncertainty remains

\footnotetext{
${ }^{1}$ Hydra and a few other animals are able to regenerate from body pieces.
} 
Table I. Recent milestones in the concepts of aging ${ }^{a, b, c}$

\begin{tabular}{ll}
\hline Decade & \multicolumn{1}{c}{ Milestone } \\
\hline 1950 s & Caloric restriction found to extend the life span of some animals \\
1950 s & Free radical damage to macromolecules thought responsible for aging \\
1960 s & Aging thought to be a biological process distinct from the pathologies associated with it \\
1974 & National Institute of Aging (USA) established indicating the need for biomedical research in aging \\
1978 & Telomeres identified at chromosome ends ${ }^{d}$ \\
$1990 s$ & Epigenetic changes recognized as heritable environment influence on genome \\
$1990 s$ & Lifespan of worms, yeast, and flies extended by gene knockout technology \\
2000 & Inflammaging; chronic inflammation contributes to aging \\
$2000 s$ & Resveratrol shown to activate sirtuins to extend the lifespan of yeast, \\
2009 & Calorie restriction in elderly humans shown to improve memory, increase insulin sensitivity, and reduce inflammatory \\
& activity
\end{tabular}

${ }^{\mathrm{a}}$ Campisi and Vijg, 2009, ${ }^{\mathrm{b}} \mathrm{Ljubuncic}$ and Reznick, 2009, ${ }^{\mathrm{C}} \mathrm{Kim}, 2007,{ }^{\mathrm{d}}$ Blackburn and Gall, 1978, ${ }^{\mathrm{e}}$ Witte et al., 2009.

Table II. Contemporary theories of aging

\begin{tabular}{|c|c|c|}
\hline Theory & Concept & Year \\
\hline Rate of living/oxidative damage theory & $\begin{array}{l}\text { Lifespan and metabolic rate are inversely correlated } \\
\text { i.e., "live fast - die young" }\end{array}$ & Pearl, 1928 \\
\hline Mutation accumulation theory & $\begin{array}{l}\text { Natural selection does not favor old age. Mutations that } \\
\text { are detrimental only in the old are not under selective } \\
\text { pressure. }\end{array}$ & Medawar, 1952 \\
\hline Free-radical theory & $\begin{array}{l}\text { Macromolecular damage is a major cause of aging. } \\
\text { Free radicals are the main source of damage. } \\
\text { Mitochondria are the major source and target of free } \\
\text { radicals. }\end{array}$ & Harman, 1956 \\
\hline Antagonistic pleiotropic model & $\begin{array}{l}\text { Genes that are beneficial at a young age may be } \\
\text { harmful at later ages. }\end{array}$ & Williams, 1957 \\
\hline Somatic mutation theory & $\begin{array}{l}\text { Aging is determined by mutations to genes after they } \\
\text { are inherited. Not all mutations can be repaired. } \\
\text { Chromosomes become inactivated. }\end{array}$ & Szilard, 1959 \\
\hline Telomere loss theory & $\begin{array}{l}\text { Telomere length and telomerase activity govern } \\
\text { proliferative potential of cells. Telomere loss } \\
\text { eventually results in senescence. }\end{array}$ & Olovnikov, 1973 \\
\hline Disposable soma theory & $\begin{array}{l}\text { Unrepaired macromolecular damage is a major cause } \\
\text { of aging. Balance is needed between resource } \\
\text { investment in somatic maintenance and repair, or in } \\
\text { reproduction. }\end{array}$ & Kirkwood and Holliday, 1979 \\
\hline
\end{tabular}

concerning the types of damage that are most important to the aging process and how damage causes cell aging.

Other concepts of aging emphasize the importance of the cellular response to damage rather than the damage itself. Transgenic mice with constitutively heightened cellular responses to damage (apoptosis and senescence) displayed phenotypes associated with aging that included osteoporosis and organ atrophy as well as shortened life span (Campisi and Vijg, 2009). Equally important, transgenic mice that over-express catalase (targeted to mitochondria) demonstrated a significant increase in life span (Shriner et al., 2005).

Environmental factors that damage cellular macromolecules or interfere with repair processes will affect aging. Included among such factors are chronic inflammatory and infectious diseases, chemicals that support oxidative stress, ultraviolet radiation, among others.

This review will address our current understanding of how and why we age. The focus will be on the effects of environmental (i.e., non-genetic) factors on the aging process. Emphasis will be given to both the nature of potent environmental agents and the mechanisms by which such agents exert their influence.

\section{PHYSICAL AGING}

The post-reproductive period is thought to be the start of physiological decline with age-related changes occurring 
Table III. Age-related structural and functional changes in major organs and organ systems

\begin{tabular}{|c|c|c|}
\hline Organ/System & Major changes in structure & Change in function \\
\hline Heart, blood vessels & $\begin{array}{l}\text { Left ventricular hypertrophy, heart walls and } \\
\text { valves thicken; blood vessel walls thicken; } \\
\text { myocytes decrease in number }\end{array}$ & $\begin{array}{l}\text { Heart rate may slow; arrhythmias increase; blood } \\
\text { pressure may increase; orthostatic hypotension } \\
\text { may occur }\end{array}$ \\
\hline Lungs & Alveoli and capillaries decrease in number & $\begin{array}{l}\text { Tissue elasticity decreases; lung function (i.e., FEV, } \\
\text { FEF, TLC) decreases }\end{array}$ \\
\hline Kidney & $\begin{array}{l}\text { Nephrons decrease in number; blood vessels } \\
\text { stiffen }\end{array}$ & Filtration may be slowed \\
\hline Musculo-skeletal & $\begin{array}{l}\text { Muscle mass decreases; bone marrow and } \\
\text { mineral content decrease; articular surface } \\
\text { frays and softens; proteoglycan monomers } \\
\text { decrease in size and aggregation capacity }\end{array}$ & $\begin{array}{l}\text { Joints stiffen; osteoarthritis and osteoporosis may } \\
\text { develop }\end{array}$ \\
\hline Immune & $\begin{array}{l}\text { Thymus atrophy; shift from naïve } \mathrm{T} \text { and } \mathrm{B} \text { cells } \\
\text { toward memory cells; reduction in bone } \\
\text { marrow stem cell output }\end{array}$ & $\begin{array}{l}\text { Decreased T cell function; decreased ability to fight } \\
\text { disease and prevent tumor development; increased } \\
\text { risk of infection }\end{array}$ \\
\hline Skin & $\begin{array}{l}\text { Epidermis thins, melanocytes increase in size, } \\
\text { decrease in number; blood vessels become } \\
\text { more fragile; subcutaneous fat decreases }\end{array}$ & $\begin{array}{l}\text { Development of elastosis, wrinkles, dryness, itching, } \\
\text { telangiectasia; increased pigmentation }\end{array}$ \\
\hline Endocrine system & $\begin{array}{l}\text { Decline in testosterone, estrogen, growth } \\
\text { hormone, and insulin-like growth factor } 1\end{array}$ & $\begin{array}{l}\text { Numerous age-related metabolic and reproductive } \\
\text { changes }\end{array}$ \\
\hline Brain and nervous system & $\begin{array}{l}\text { Decreased brain cellularity; cell axons fray, } \\
\text { decreased signal transmission; neural network } \\
\text { disruptions }\end{array}$ & Decreased cognitive function \\
\hline
\end{tabular}

FEF: forced expiratory flow, $\mathrm{FEV}_{1}$ : forced expiratory volume in 1 second, TLC: total ling capacity.

in appearance and function. The changed appearance has been referred to as the aging phenotype. According to the Disposable Soma theory, it is the time when the "soma has survived long enough to be disposable" (Kirkwood, 2005). With age, structural and functional changes occur in most organs. Those organ changes that have major effects on health are listed in Table III and discussed below.

\section{Heart, blood vessels, blood}

With aging, major changes occur in the anatomy and physiology of the heart. The walls of the left ventricle thicken allowing more blood to be pumped into stiffer blood vessels. The heart rate is slower in the elderly. The maximum heart rate in response to exercise is decreased. The body's ability to perform vigorous exercise declines by about $50 \%$ between the ages of $20-80$ years (Joyner, 2008). There is a reduction in the number of myocytes and those that remain enlarge, compromising their ability to pump blood efficiently.

The aorta and other arteries become thicker and stiffer with age. Capillary walls thicken slightly. These changes result in increased blood pressure (Joyner, 2008).

Hematopoietic changes occur as evidenced by an increase in myeloproliferative disease, in declining adaptive immunity, and in greater propensity to anemia (Warren and Rossi, 2009). The normal decrease in body water con- tent results in less fluid in the bloodstream and a decrease in blood volume. Hematopoietic stem cells show increased propensity to differentiate toward myeloid rather than lymphoid lineages. This may contribute to the decline in lymphopoiesis that accompanies aging. These changes would normally have little consequence on heart performance, but the aging heart would consequently be less able to respond to increased workloads that might result from illness, infections, and certain medications.

\section{Lung}

The lung begins to lose tissue after age twenty. The number of alveoli decreases as does the amount of lung capillaries. The lungs become less elastic. With age, the diaphragm and intercostal muscles become weaker. Maximum lung function decreases, with reduced maximal inspiratory and expiratory forces. A change in breathing pattern may develop to compensate for decreased ability to expand the chest.

Other respiratory changes may make the elderly more susceptible to pneumonia and other lung infections. The airways in the elderly tend to close more readily and may collapse upon shallow breathing, thereby increasing the risk of lung infection. Additionally, the cough reflex may not trigger as readily and the cough itself may be less forceful. Airway cilia are less able to move mucus up and out of the 
airway. Ordinarily, low oxygen levels or high carbon dioxide levels trigger an increased rate and depth of breathing. With age, this response is reduced. Chronically low oxygen levels also contribute to the increased risk of bronchitis or pneumonia in the elderly.

\section{Kidney}

The kidney functions to remove waste and excess water from the blood to form urine. Many substances become more concentrated in the urine relative to the blood. With advancing age, the concentrating ability of the kidney decreases requiring a greater volume of water to excrete the same amount of waste material. The reduction in renal function is due in part to loss of nephrons, to reduced activity of cellular enzymes, and to a lessening of blood flow to the kidney. However, since the kidney receives a relatively great excess of blood, its reduced functioning normally does not result in an accumulation of waste products in the blood.

\section{Musculo-skeletal}

Osteoarthritis, a degenerative cartilage disease, is the most common disease of aging and aging is the principal factor responsible for the disease. It is thought that the disease begins with mechanical stress on the superficial cartilage layer, followed by deterioration of the layer (Horton et al., 2006). The disease progresses to remodeling and degradation of the cartilage extracellular matrix. The process is irreversible and results in loss of the underlying cartilage layers. Eventually, bone begins to grind against bone, causing pain and inflammation (Taniguchi et al., 2009).

\section{Endocrine system}

Aging causes changes in endocrine tissue activity. Endocrine tissues may produce less hormone and target tissues may become less responsive to their controlling hormone. Additionally, hormones may be metabolized more slowly. In women, estrogen and prolactin levels typically decrease; in men, testosterone levels decrease. With aging, endocrine tissue may produce less hormone, or it may produce the same amount at a slower rate. Since endocrine organs may be controlled by other hormones, the amount of regulating hormone may stay the same, but the response by the endocrine organ may change with aging.

\section{Immune system}

Overall, immune function declines with age with the innate immune system being better preserved than the adaptive compartment. The thymus, the site of T cell maturation, begins to atrophy after adolescence and by middle age is reduced to $15 \%$ of its maximum size. This results in an age-dependent decline in the absolute number of $T$ cells. Changes in gene expression of hematopoetic stem cells skews their differentiation towards reduced lymphoid progenitors thus contributing to a decrease in the production of naïve $T$ and $B$ cells. The shift toward antigenexperienced memory cells, and away from naïve cells, supports the declining response to new antigenic challenges (viral, bacterial, neoplasms). The result is increased susceptibility to infectious diseases and increase in cancer occurrence.

An age-dependent increase of type 1 (IL-2, IFN-gamma, TNF-alpha) and type 2 (IL-4, IL-6, IL-10) positive CD8+ T-cells indicates up-regulation of inflammatory responses (Sansoni et al., 2008). This heightened pro-inflammatory state, referred to as inflammaging (discussed in a later section) is detrimental to longevity by contributing to agerelated inflammatory diseases including osteoporosis, arteriosclerosis, and dementia.

\section{Skin}

The skin can display visible signs of aging that include wrinkles, pigmentation and sagging. It has been estimated that more than $90 \%$ of all elderly people have some type of skin disorder. Exposure to ultraviolet light prematurely ages the skin and is responsible for the changes noted above.

The epidermis thins with aging. The number of melanocytes decreases, but the remaining melanocytes increase in size. Pigmentation may increase, especially in sun-exposed areas of the skin. Degeneration of the elastic tissue, i.e., elastosis, may also be pronounced in sun-exposed areas. With aging, blood vessels (in the dermis) become fragile, which may lead to bruising, bleeding under the skin, and cherry angiomas.

Sebaceous glands produce less oil, and sweat glands produce less sweat with aging. The subcutaneous fat layer thins, reducing the ability to maintain body temperature. Some topically-applied medications are absorbed by the fat layer, and loss of this layer changes the absorption kinetics.

\section{Brain}

Autopsy studies have shown brain mass increases during childhood and adolescence, decreases slowly through adulthood, then, decreases rapidly after the age of 80 years (Rushton and Ankney, 1996). Over time, bundles of axons (white matter) become frayed thereby impairing communication among brain regions. There is a loss of both gray matter (nerve cell bodies and supporting cells) 
and neurotransmitters with age. Deteriorating nerves and nerve endings result in a reduced sense of taste and smell.

Neurodegeneration, the deterioration of neurons and their myelin sheaths, begins before symptoms are noted. Usually, there is a progressive decline in cognitive function during mid-life (Valdes et al., 2008). Only when numerous cells die, or cease to function, do symptoms become apparent. The dysfunction and disabilities that result are referred to as neurodegenerative disease.

Each neurodegenerative disease (i.e., Parkinson's disease, Alzheimer's disease) has a separate etiology that typically involves both genetic and environmental factors. Among the environmental factors, oxidative stress is thought to be a central process whereby reactive oxygen and reactive nitrogen species damage neural membranes, i.e., Free radical theory of aging. The result is a decline in cognitive and motor performance.

\section{THE MOLECULAR BIOLOGY OF AGING}

After mammals attain reproductive maturation, there is a gradual physiologic decline. Although impacted by the environment, the aging process is largely under genetic control. The genes that control longevity do so by addressing cell maintenance and repair. A database of genes related to aging and longevity of both humans and model systems, GenAge, has been created and is available at http://genomics.senescence.info/genes. As of 20 July 2008, the database contained 261 human genes, 789 genes in model organisms, and 258 longevity-associated human genes (genes linked to a mechanism or pathway associated with longevity). An analogous database, AnAge, is comprised of longevity and aging genes found in animal species. As of April 2008, AnAge included 1,331 mammals and 4,122 entries on aging and longevity. These databases have proven to be invaluable to researchers probing the molecular genetics of human aging.

As a result of damage to cellular DNA, RNA, proteins or membranes, cells may undergo senescence. This irreversible arrest of the cell cycle prevents damaged cells from proliferating and expressing deleterious mutations such as those responsible for malignant tumors. Although senescence prevents cancers, it may also contribute to disease. Senescent cells may release enzymes, growth factors or cytokines that may cause neighboring cells to proliferate (Blagosklonny and Camprisi, 2008). In culture, senescent fibroblasts were found to alter the local tissue environment to promote the growth of preneoplastic epithelial cells (Parrinello et al., 2005). It is possible then that changes in the microenvironment caused by senescent cells can create a local tissue environment that promotes the growth and progression of preneoplastic cells toward malignancy.

\section{Epigenetics}

Environmental influences on the genome extend beyond the DNA sequence. Heritable changes in gene expression that do not involve changes to the DNA sequence are referred to as epigenetic changes. Such changes can be made to the DNA or to the protein. Analogous to the genome, this heritable system is referred to as the epigenome, because the changes are inherited across cell division even though they are not encoded in the DNA. Although genomic information is uniform among the different cells of an organism, the epigenome varies from tissue to tissue, controlling the expression of genes. Examples of epigenetic modifications are DNA methylation and histone acetylation. Transcription factors recognize the epigenetic marks and perform the tasks indicated by the mark.

Epigenesis is an ongoing, lifelong process in which the epigenetic state can change by interaction with elements in either or both the internal (eg, hormones, growth factors) or external (eg, diet, oxidative agents) environments (Fraga et al., 2005). Epigenetic changes can be modified by the environment, and therefore are considered to be the site where the environment meets the genome. The impact of the environment on the epigenome was dramatically demonstrated by Fraga et al. (2005) who examined the global and locus-specific differences in DNA methylation and histone acetylation of a large cohort of monozygotic twins. They found that twins were epigenetically indistinguishable during the early years of life, whereas older monozygous twins displayed remarkable differences in their overall content and genomic distribution of 5-methylcytosine DNA and histone acetylation, affecting their gene-expression. Prominent among the external factors that have been proposed to have a long-term influence on epigenetic modifications are smoking, physical activity, diet, oxidative stress, hormones, and environmental toxicants (Kahn and Fraga, 2009). Epigenetic modification is one of the pathways by which the environment exerts a profound effect on the aging process and the human lifespan (Fig. 1 for other environmental factors that impact aging and lifespan).

The mechanisms that account for epigenesis are not known. DNA methylation usually decreases with increasing age, and is often associated with silencing of genes. However, a number of specific loci become hypermethylated during aging, including some tumor suppressor genes (Kahn and Fraga, 2009). Histone modification can be associated with gene activation and gene repression. Other 


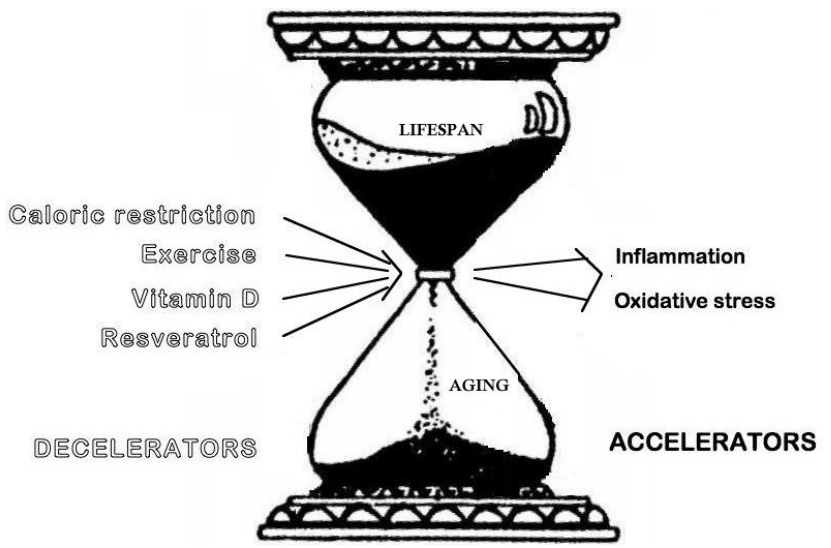

Fig. 1. Environmental factors that influence the aging process. Lifespan is depicted as sand passing through the aging process. The time to complete the process is hastened by inflammation and oxidative stress (accelerators) shown as widening the neck of the hourglass. The process is slowed and lifespan extended by factors such as caloric restriction, exercise, vitamin $\mathrm{D}$ and resveratrol (decelerators) which constrict the hourglass neck.

mechanisms that play a role in epigenetic regulation include telomere shortening and protein deacetylation (via sirtuins) as discussed below.

\section{Chromosomal stability}

Located at the ends of chromosomes, telomeres are repetitive DNA-protein complexes that protect the chromosomes from degradation, thereby promoting chromosomal stability. With each cell division, telomeres shorten, eventually sending cells into senescence. In humans, telomere length is maintained by telomerase, an enzyme that adds repeat DNA sequences to the chromosomal ends, thus preserving both telomere length and cell function. With age, however, telomerase production decreases resulting in the shortening of telomeres.

Telomere length has been proposed as a biomarker of aging. In roundworms, telomere length has been correlated with lifespan (Joeng et al., 2004). Worms with longer telomeres were found to live about 20 percent longer than normal worms. Shortened telomeres may cause chromosomal instability and have been associated with age-related diseases including dementia and other cognitive declines (Martin-Ruiz et al., 2006; Starr et al., 2008). Shortened telomeres were found to predict poor clinical outcomes from several chronic diseases including: mortality in patients with stable coronary artery disease (FarzanehFar et al., 2008); increased risk of metastasis in patients with breast cancer; increased risk of bladder, head and neck, lung, and renal-cell cancers; and worse progression

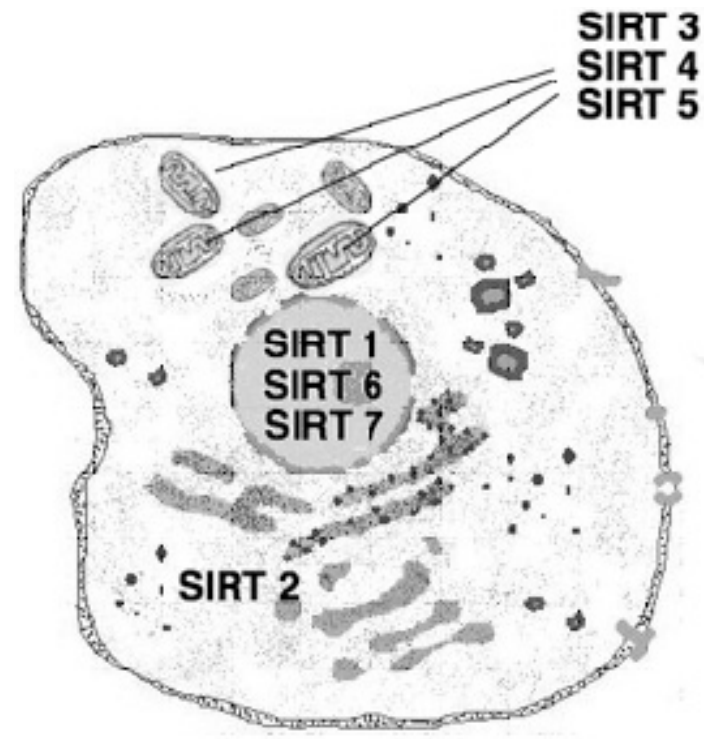

Fig. 2. Cellular location of the seven human sirtuin homologs. SIRT1, SIRT6 and SIRT7 reside in the nucleus where SIRT 1 is normally bound to DNA. SIRT2 is located in the cytoplasm; SIRT3, SIRT4 and SIR5 are found in the mitochondria where they function in energy metabolism.

in patients with colorectal cancer (Ornish et al., 2008). Of considerable interest, though, is a study that showed "super" seniors (exceptionally healthy individuals aged 85 years or older) did not have unusually long telomeres for their age. Rather, they had lower variability in telomere length compared with mid-life controls (Halaschek-Wiener et al., 2008).

In humans, systemic telomere length (usually measured in peripheral leukocytes) appears to be determined predominantly by genetics with some contribution from environmental factors. Among the non-genetic influences, oxidative stress and inflammation have been found to accelerate telomere shortening (De Meyer et al., 2008). Oxidative stress appeared to down-regulate telomerase activity in cultured vascular smooth muscle cells and endothelial cells (Ornish et al., 2008). Mice that lacked telomerase activity displayed increased oxidative stress and reduced catalase activity, indicating a redox imbalance (PérezRivero, 2008). Cultured fibroblasts from telomerase deficient mice had greater oxidant damage (higher superoxide anion and hydrogen peroxide production) and lower catalase activity. Restoration of telomerase activity produced increased catalase expression and activity.

Inflammation has been cited as a contributor to telomere attrition (Carrero et al., 2008). Environmental factors have also been shown to increase telomerase activity. Telomerase activity was $30 \%$ greater in subjects who had made 
lifestyle changes that included a low-fat diet, regular exercise and stress reduction (Ornish et al., 2008). Although not measured, increased telomere length is implied from the increase in telomerase activity.

\section{Sirtuins}

Sirtuins, silence information regulators (SIRs), discovered in 1987, are a family of protein deacetylases that are found in organisms ranging from bacteria to humans (Gan and Mucke, 2008). Located in the cell nucleus, mitochondria, or cytoplasm (Fig. 2), sirtuins regulate cell functions by deacetylating both histone and nonhistone targets. When upregulated, they appear to support and promote longevity and can extend life span.

The sirtuins contain a conserved catalytic core conferring NAD+-dependent protein deacetylase and ADP-ribosyltransferase activity (Ford et al., 2006). Being dependent on the relative levels of NAD+ and NADH, they are directly linked to the energy status of the cell.

There are seven known sirtuin homologs in humans (Table IV). The sirtuins that reside in the nucleus (i.e, SIRT1, SIRT6, and SIRT7) have been implicated in genomic stability and cell proliferation. SIRT1, normally bound to DNA, is involved in chromosome maintenance, and contributes to the silencing of many gene promoters (Vijg et al., 2008). In response to DNA damage and oxidative stress, SIRT1 deacetylates p53, repressing p53-dependent apoptosis. As DNA damage accumulates, SIRT1 proteins are called upon to address DNA repair, thus limiting their ability to perform chromatin maintenance.

Activation of SIRT1 has been found to delay the aging process (Gan and Mucke, 2008) and overexpression of SIRT1 was shown to protect against neurodegeneration (Kim et al., 2007).

In one study, lab mice with excess SIRT1 showed both improved ability to repair DNA and a lifespan extended by 46 per cent (Sinclair, 2005). As detailed in a later section of this review, SIRT1 is linked to calorie restriction, a dietary regimen that slows aging in mammals.
Located in the cytoplasm, SIRT2 is involved in mitosis and differentiation. SIRT3, SIRT4, and SIRT5 are found in mitochondria where they function in energy metabolism and response to oxidative stress. Sirtuin-mediated deacetylation is important in the maintenance of mitochondrial function during aging. Both mitochondrial oxygen radical production, and the level of fatty acid unsaturation in the mitochondrial cellular membranes contribute to the aging process (Kim et al., 2007).

SIRT6 is involved with genomic stability and protection of telomeres. In mice, inactivation of SIRT6 leads to a premature aging phenotype and a dramatically shortened life span (Kriete and Mayo, 2009). SIRT6 binds to a subunit of NF-kappa B thereby restraining the activity of the transcription factor. NF-kB controls the activity of genes involved in apoptosis, cell senescence, inflammation, and immunity. Activation of NF-kB has been found to induce insulin resistance, muscle atrophy and age-associated morbidities (Ljubuncic and Reznick, 2009). Blockade of NF-kB reversed the gene expression and skin characteristics of aged mice to that of younger animals (Kawahara et al., 2008). Mice that lacked SIRT6 had elevated levels of NF-kB-dependent genes and within four weeks of birth had died from an aging-like condition (Adler et al., 2008).

A number of environmental factors have been found to activate sirtuin pathways. Prominent among them are resveratrol (3,4',5-trihydroxystilbene found in grapes and red wine) and calorie restriction. These factors, and others, are discussed in a later section.

\section{Oxidative stress}

Oxidative stress appears to be one of the major environmental factors contributing to physiological aging, age-related diseases, and reduced lifespan (Fig. 1). Oxidative stress refers to an imbalance between oxidants and antioxidants, in favor of the oxidants, that potentially leads to cellular damage. Prominent among the endogenous oxidants are reactive oxygen species (ROS) including superoxide anions, hydroxyl radicals, and hydrogen peroxide.

Table IV. Human sirtuins location and function ${ }^{\mathrm{a}}$

\begin{tabular}{clll}
\hline Sirtuin & Intracellular location & Activity & Function \\
\hline SIRT1 & Nucleus & Deacetylase & Metabolism, DNA repair \\
SIRT2 & Cytoplasm & Deacetylase & Cell cycle, tumorigenesis \\
SIRT3 & Nucleus, mitochondria & Deacetylase & Metabolism \\
SIRT4 & Mitochondria & ADP-ribosyl transferase & Insulin secretion \\
SIRT5 & Mitochondria & Deacetylase & Unknown \\
SIRT6 & Nucleus & Deacetylase & Telomere maintenance \\
SIRT7 & Nucleus & ADP-ribosyl transferase & DNA repair \\
\hline
\end{tabular}

${ }^{a}$ Modified from North and Verdin, 2004. 
The effects from excess intracellular oxidants include damage to cellular components and activation of cellular signaling pathways such as NF-kB signaling, p53 activation, heat shock response among others (Finkel and Holbrook, 2000).

Reactive oxygen species can be generated exogenously or produced intracellularly. The majority of the intracellular ROS is derived from the mitochondria. Because of its proximity to the main source of oxidant production, mitochondrial DNA is thought to be highly susceptible to oxidative damage. Results of such damage to mitochondrial DNA is mitochondrial dysfunction with ensuing production of more ROS, leading to further DNA damage.

Increased ROS and the corresponding response to oxidative stress are considered to be major factors in determining longevity. The free-radical theory of aging (Table II) proposes that endogenous oxygen radicals result in cumulative damage and eventual death. According to the theory, the higher the metabolic rate of an organism, the greater the production of reactive oxygen species, and the shorter the life span.

Factors that favor resistance to stress should extend life span. In transgenic mice, genes found to be life-extending included those regulating energy use, and the level of ROS (Campisi and Vijg, 2009). In drosophila, strains of flies with extended life span had increased resistance to oxidative stress that was associated with enhanced antioxidant enzyme activity (Harshman and Haberer, 2000).

\section{Oxidants, inflammation, and age-related diseases}

The incidence of cardiovascular and neurodegenerative diseases increases with age. A common feature of these conditions is involvement of inflammatory cells. These cells contribute to oxidative stress via the NADPH oxidase complex, which produces large amounts of superoxide. Superoxide can interact with endogenously generated nitric oxide leading to the formation of peroxynitrite and other radical species. Reduced oxidative stress is also thought to be involved with the increased longevity associated with calorie restriction, as will be discussed on a later section of this review.

\section{Inflammation}

Centenarians (i.e., those individuals who reach the age of 100 years or greater) are characterized by having an anti-inflammatory genetic background. They possess a high frequency of genes associated with control of inflammation, for example, genes that control IL (interleukin)-4, -10 , and -13 . This genetic background appears to protect them from age-related inflammatory diseases, such as atherosclerosis and cardiovascular disease, thereby increasing their chance of becoming centenarians (Vasto et al., 2008).

Inflammation refers to a localized response to injury that functions to reduce, or wall off, both the injurious agent and the injured tissue. Chronic inflammation is systemic, persistent, low-grade inflammation that is accompanied by systemic up-regulation of pro-inflammatory factors. Chronic inflammation has been linked to age-related chronic diseases such as osteoarthritis and Alzheimer's disease. This association between chronic inflammation and aging has been termed inflammaging (Franceschi et al., 2000). Studies have shown that aging is characterized by low grade systemic inflammation, and moreover, that inflammatory markers are predictors of mortality in the elderly (Vasto et al., 2008). However, though inflammaging may be a risk factor for many age-related pathologies, it is not clear if it is a cause or an effect of aging (Giunta et al., 2008).

How does chronic inflammation promote aging? The inflammatory response involves an increase in leukocyte replication. The relationship between inflammation, leukocyte telomere length (LTL), and aging has been addressed in several studies (Epel et al., 2004; Demissie et al., 2006; Fitzpatrick et al., 2007). LTL was found to be relatively short in those with chronic inflammation, and was an independent risk factor for coronary heart disease. Thus, shortened LTL may be a marker of chronic inflammation and aging-related diseases.

The paradox of inflammaging is that strong acute inflammatory responses during childhood and early adulthood contribute to fitness and survival. If however, the inflammatory response persists, i.e., becomes chronic, the individual becomes susceptible to disease. Is there a tradeoff between early beneficial effects and late negative outcomes as suggested by Franceschi et al. (2000) Both acute and chronic stages of inflammation are influenced by genetic components and environmental factors.

\section{ENVIRONMENTAL FACTORS MODULATE THE RATE OF AGING}

Life expectancy is continually increasing. In the developed world, it is increasing at a rate of five or more hours per day (Kirkwood, 2008). Today, a person can live to 120 years. Longevity is determined by the status of all the molecules present at and after the time of reproductive maturation. Numerous factors have been associated with cellular damage and repair. Those that have been shown to influence the aging process are discussed below. 


\section{Caloric restriction (CR)}

Diet is a key environmental factor that affects the incidence of chronic diseases. Although reports of beneficial effects from dietary supplements are frequent, currently the greatest interest in diet as it relates to aging is the demonstrated association of caloric restriction with longevity.

Caloric restriction, the reduction in caloric intake while maintaining intake of essential nutrients, appears to be a non-genetic (i.e., environmental) means to extend life span. Caloric restriction has been found to extend the lifespan of species as diverse as yeast, drosophila, rhesus monkeys, and humans. Restricting calories to $30-50 \%$ less than an ad libitum animal would consume while maintaining proper nutrient intake, has been found to increase the lifespan of mice up to $50 \%$ while decreasing the frequency of age-related neurodegenerative diseases and a delay in onset of cancer (Shumizu and Shirasawa, 2008).

Resveratrol (3,4',5-trihydroxystilbene), a SIRT1 agonist, was found to extend the life span of mice that had been fed a high-caloric diet (Baur et al., 2006), to reduce neuronal loss, and to improve associative learning (Kim et al., 2007). Resveratrol is a polyphenolic compound found in grapes, red wine, peanuts, and other foods. When taken orally, it is absorbed, then rapidly metabolized and eliminated. Resveratrol activates SIRT1 thus restoring chromosomes, reducing insulin resistance, increasing mitochondrial functions, and prolonging survival in mice. Putting animals on reduced-calorie diets for a couple of weeks dramatically slowed cell proliferation rates (Varady et al., 2008). Thus calorie restriction may reduce the risk of cancer in animals by slowing the growth of abnormal cells.

Intermittent fasting and calorie restriction have also been shown to reduce cognitive decline in animals. Caloric reduction in humans appears to have a similar effect. Reducing calories $30 \%$ per day increased the memory function of elderly men and women (Witte et al., 2009). Scientists examined health data from Mormons, who typically don't smoke or drink alcohol, and frequently abstain from food on the first Sunday of every month. When compared with most Americans, the researchers found Mormons had lower rates of heart disease, and after controlling for factors that protect against heart disease, they found that only fasting made a significant difference in lowering the risk of heart disease. More than a $40 \%$ reduction in heart disease risk was noted, as well as a lower incidence of diabetes (Horne et al., 2008).

Health consequences from fasting were also studied in asthma patients. Patients who fasted were found to have fewer symptoms, better airway function, and a decrease in markers of inflammation in the blood, when compared with those who didn't fast or restrict calories (Car and Sheikh, 2004).

In animals, calorie restriction has been shown to enhance immune responses, to stimulate DNA repair systems, and to better withstand environmental assault from infectious agents, toxins, radiation, and extreme temperatures (Masoro, 2000). It appears also to delay onset of chronic visceral and brain diseases (Longo and Finch, 2003).

Several theories have been proposed to explain the mechanism(s) underlying the multitude of effects of caloric restriction. As indicated in Table II, the free-radical theory of aging links metabolism with aging since oxygen free radicals are a by-product of oxidative phosphorylation. The accumulation of free radical-damaged molecules would lead to decreased cellular activity, and ultimately to the death of the organism. This theory has been expanded to the oxidative damage theory to include reactive oxygen species that are not free radicals, but may also cause molecular damage (Bys et al., 2007). Caloric restriction may reduce the production of both reactive oxygen species and reactive nitrogen species thereby suppressing the activation of redox-sensitive transcription factors. Caloric restriction reduces glycolysis and increases oxidation of lipids and amino acids, thereby diminishing the production of reactive oxygen species in the mitochondria.

One hypothesis for calorie restriction proposes that it acts by decreasing oxidative stress. In support of this hypothesis, the rate of oxidant generation of mitochondria from calorically restricted mice is significantly lower than from their ad libitum-fed counterparts, and caloric restriction reduces the age-associated accumulation of oxidatively damaged proteins, lipids and DNA (Finkel and Holbrook, 2000).

Other theories are based on caloric restriction-induced increased expression of cytoprotective and restorative proteins including growth factors, anti-oxidant enzymes, and protein chaperones such as heat shock proteins. Caloric restriction modifies acyl composition of neural membrane bilayers and is associated with decreased membrane lipid peroxidation and lifespan extension.

\section{Vitamin D}

Vitamin D deficiency is common in the elderly and is thought to result both from reduced sun exposure and reduced ability of the skin to produce the vitamin. In the elderly, vitamin $D$ deficiency is associated with increased risk of bone fracture, and of type 2 diabetes among other chronic diseases. Low serum levels of vitamin $\mathrm{D}$ have also been associated with cognitive impairment (Llewellyn et 
al., 2009).

Mechanistically, vitamin D appears to inhibit inflammation. With aging, vitamin $\mathrm{D}$ concentrations decrease, whereas inflammatory mediators increase. Vitamin $D$ deficiency was associated with elevated levels of C-reactive protein and interleukin-10, both markers of systemic inflammation (Lee et al., 2008). Administration of calcitriol (vitamin D3) to vitamin D-deficient individuals resulted in a down-regulation of inflammatory markers, including C-reactive protein (Lips, 2006).

Vitamin $D$ has also been associated with modulation of telomere length. In a group of 2160 women (18-79 years; mean age 49.4) serum leukocyte telomere length (LTL) was negatively correlated with age $(r=-0.40, p<0.0001)$, but positively associated with serum vitamin $D$ concentrations ( $r=0.07, p=0.0010$ ) (Ayrun et al., 2007; Richards et al., 2007). The latter association persisted after adjustment for age, season of vitamin $D$ measurement, menopausal status, use of hormone replacement therapy, and physical activity. The positive association between higher vitamin $D$ concentrations and telomere length is equivalent to 5 years of telomeric aging. These results suggest beneficial effects of vitamin $D$ and possibly attenuation of LTL degradation.

Low blood levels of vitamin $D$ have been associated with an increased risk of dementia (Llewellyn et al., 2009). In a group of 1,766 people over age 65 , blood levels of vitamin $D$ were measured, and mental function was assessed (by questionnaire). About $12 \%$ of the individuals were found to be cognitively impaired. Serum vitamin $D$ levels were found to be generally lower in the cognitively impaired population, and the lower their vitamin $\mathrm{D}$ level, the more likely impaired individuals were in that group. After adjusting for age, sex, education and ethnicity, those in the lowest quartile for serum vitamin D were 2.3 times more likely to be impaired compared with those in the highest quarter (Llewellyn et al., 2009).

The role of vitamin $D$ in cognitive function is unclear. Vitamin $D$ receptors are present on neurons and glial cells, and genes encoding the enzymes involved in the metabolism of calcitriol are expressed in brain cells. Based on these findings, the importance of proper nutrition for the elderly is undeniable.

\section{Heavy metals}

Acute exposure to heavy metals may cause acute neurotoxicity. Slow accumulation can result in neurodegenerative disease later in life. Mercury and lead have been implicated in the etiology of neurodegenerative diseases (Monnet-Tschudi et al., 2006). Experimentally, continuous application, for 10-50 days, of non-cytotoxic concentrations of these heavy metals resulted in their accumulation in fetal rat brain cells in culture and in the occurrence of delayed toxic effects. Mercury and lead have been found to induce glial cell reactivity (a hallmark of brain inflammation) and to increase the expression of the amyloid precursor protein. Mercury also stimulates the formation of insoluble beta-amyloid, which appears to play a prominent role in Alzheimer's disease. Lead and mercury may also induce apoptotic cell death (Monnet-Tschudi et al., 2006).

Mechanistically, the heavy metals have pro-oxidant activity and have been found to exacerbate the age-related increase in oxidative stress mentioned earlier. They also appear to contribute to the decline of antioxidant defenses.

\section{Exercise}

Exercise is believed to have many anti-aging benefits. Regular exercise has been reported to increase mean lifespan, to reduce the risk of developing neurological disorders, and to delay the onset of neurodegenerative disease (Larson et al., 2006). Exercise elevates high-density lipoproteins (HDL). In addition to scavenging cholesterol, these lipoproteins appear to have anti-oxidant and anti-inflammatory activities.

Exercise has been found to increase acute oxidative stress, even in conditioned athletes. It is thought that some of its benefits may be a consequence of a stress-tolerance mechanism (Finkel and Holbrook, 2000).

\section{CONCLUSIONS}

Aging is a progressive process that is driven by accumulated molecular damage and regulated by systems of maintenance and repair. Aging does not appear to be a result of evolutionary selection. Aging is frequently considered in terms of mechanisms that lead to decline in function, i.e., epigenetic modifications, telomere shortening, oxidative stress, and chronic inflammation. Complex biological mechanisms that are driven by genetic and environmental pressures guide the aging process.

GenAge, the catalogue of genes associated with aging and longevity, has been invaluable in identifying the basic aspects of the aging process. The major gene classes associated with aging are: the stress response, biosynthesis, protein metabolism, and energy metabolism. The genes therein have been shown to be responsive to environmental influences.

Chemical damage to macromolecules that goes unrepaired is a major cause of aging. Animals in which damage accumulates at an accelerated rate, or which are 
made deficient in antioxidant defense genes, show premature aging phenotypes and a shortened lifespan. Diverse environmental stimuli can perturb the redox balance toward oxidative stress. Among the most important of these stimuli are UV radiation, infections and heavy metals.

Can aging be retarded and lifespan extended by controlling oxidant damage and treating chronic inflammation? Transgenic mice that over-express catalase (localized to mitochondria) have shown an increase, albeit modest, in lifespan. On the other hand, overexpression of major anti-oxidant enzymes: copper zinc superoxide dismutase, catalase, and manganese superoxide dismutase (in the peroxisomes) did not show lifespan extension, thus emphasizing the importance of targeting antioxidant factors to the appropriate organelle.

Increased understanding of the biological processes responsible for aging, and the impact of lifelong environmental assaults on these processes raises the hope that medical and societal interventions will soon be able to reduce the rate, severity of damage, and functional decline associated with aging.

\section{REFERENCES}

Adler, A. S., Kawahara, T. L., Segal, E. and Chang, H. Y. (2008). Reversal of aging by NFkB blockade. Cell Cycle 7, 556-559.

Ayrun, N., Xiaobin, L., Surdulescu. G. L., Swaminathan, R., Spector, T. D. and Aviv, A. (2007). Higher serum vitamin D concentrations are associated with longer leukocyte telomere length in women. Am. J. Clin. Nutr. 86, 1420-1425.

Balaban, R. S., Nemoto, S. and Finkel, T. (2005). Mitochondria, oxidants, and aging. Cell 120, 483-495.

Blackburn, E. H. and Gall, J. (1978). A tandemly repeated sequence at the termini of the extrachromosomal ribosomal RNA genes in Tetrahymena. J. Mol. Biol. 120, 33-53.

Brys, K., Vanfleteren, J. R. and Braeckman, B. P. (2007) Testing the rate-of-living/oxidative damage theory of aging in the nematode model Caenorhabditis elegans. Exper. Gerontol. 42, 845-851.

Campisi, J. and Vijg, J. (2009). Does damage to DNA and other macromolecules play a role in aging? If so, how? J. Gerontol. A Biol. Sci. Med. Sci. 64A, 175-178.

Cappola, A. R., Xue, Q. L., Ferrucci, L., Guralnik, J. M., Volpato, S. and Fried, L. P. (2003) . Insulin-like growth factor I and interleukin-6 contribute synergistically to disability and mortality in older women. J. Clin. Endocrinol. Metab. 88, 20192025.

Car, J. and Sheikh, A. (2004). Fasting and asthma: an opportunity for building patient-doctor partnership. Prim. Care Respir. J. 13, 133-135.

Carrero, J. J., Stenvinkel, P., Fellström, B. Qureshi, A. R., Lamb, K., Heimbürger, O., Bárány, P., Radhakrishnan, K., Lindholm, B., Soveri, I., Nordfors, L. and Shiels, P. G. (2008). Telomere attrition is associated with inflammation, low
fetuin-A levels and high mortality in prevalent haemodialysis patients. J. Intern. Med. 263, 302-312.

De Meyer, T., Rietzschel, E. R., De Buyzere, M. L., Van Criekinge, W. and Bekaert, S. (2008). Studying telomeres in a longitudinal population based study. Front. Biosci. 13, 2960-2970.

Demissie, S., Levy, D., Benjamin, E. J., Cupples, L. A., Gardner, J. P., Herbert, A., Kimura, M., Larson, M. G., Meigs, J. B., Keaney, J. F. and Aviv, A. (2006). Insulin resistance, oxidative stress, hypertension, and leukocyte telomere length in men from the Framingham Heart Study. Aging Cell 5, 325-330.

Epel, E. S., Blackburn, E. H., Lin, J., Dhabhar, F. S., Adler, N. E., Morrow, J. D. and Cawthon, R. M. (2004). Accelerated telomere shortening in response to life stress. Proc. Natl. Acad. Sci., USA 101, 17312-17315.

Farooqui, T. and Farooqui, A. A. (2009). Aging: an important factor for the pathogenesis of neurodegenerative diseases. Mech. Ageing Dev. 130, 203-215.

Farzaneh-Far, R., Cawthon, R. M., Na, B., Browner, W. S., Schiller, N. B. and Whooley, M. A. (2008). Prognostic value of leukocyte telomere length in patients with stable coronary artery disease: data from the Heart and Soul Study. Arterioscler. Thromb. Vasc. Biol. 28, 1379-1384.

Finkel, T. and Holbrook, N. J. (2000). Oxidants, oxidative stress and the biology of ageing. Nature 408, 239-247.

Fitzpatrick, A. L., Kronmal, R. A., Gardner, J. P., Psaty, B. M., Jenny, N. S., Tracy, R. P., Walston, J., Kimura, M. and Aviv, A. (2007). Leukocyte telomere length and cardiovascular disease in the Cardiovascular Health Study. Am. J. Epidemiol. 165, 14-21.

Fraga, M. F., Ballestar, E., Paz, M. F., Ropero, S., Setien, F., Ballestar, M. L., Heine-Suñer, D., Cigudosa, J. C., Urioste, M., Benitez, J., Boix-Chornet, M., Sanchez-Aguilera, A., Ling, C., Carlsson, E., Poulsen, P., Vaag, A., Stephan, Z., Spector, T. D., Wu, Y. Z., Plass, C. and Esteller, M. (2005). Epigenetic differences arise during the lifetime of monozygotic twins. Proc. Natl. Acad. Sci. USA 102, 10604-10609.

Franceschi, C., Bonafe, M., Valensin, S., Olivieri, F., De Luca, M., Ottaviani, E. and De Benedictis, G. (2000). Inflammaging. An evolutionary perspective on immunosenescence. Ann. N. Y. Acad. Sci. 908, 244-254.

Gan, L. and Mucke, L. (2008). Paths of convergence: Sirtuins in aging and neurodegeneration. Neuron 58, 10-14.

Giunta, B., Fernandez, F., Nikolic, W. V., Obregon, D., Rrapo, E., Town, T. and Tan, J. (2008). Inflammaging as a prodrome to Alzheimer's disease. J. Neuroinflam. 5, 1742-1756.

Halaschek-Wiener, J., Vulto, I., Fornika, D., Collins, J., Connors, J. M., Le, N. D., Lansdorp, P. M. and Brooks-Wilson A. (2008). Reduced telomere length variation in healthy oldest old. Mech. Aging and Dev. 129, 638-641.

Harman, D. (1956). Aging: a theory based on free radical and radiation chemistry. J. Gerontol. 11, 298-300.

Harshman, L. G. and Haberer, B. A. (2000). Oxidative stress resistance: a robust correlated response to selection in extended longevity lines of Drosophila melanogaster. J. Gerontol. A 55, B415-B417.

Horne, B. D., May, H. T., Anderson, J. L., Kfoury, A. G., Bailey, B. M., McClure, B. S., Renlund, D. G., Lappè, D. L., Carlquist, J. F., Fisher, P. W., Pearson, R. R., Bair, T. L., Adams, T. D., Muhlestein, J. B. and Intermountain Heart 
Collaborative Study (2008). Usefulness of routine periodic fasting to lower risk of coronary artery disease in patients undergoing coronary angiography. Amer. J. Cardiol. 102, 814-819.

Horton, Jr. W. E., Bennion, P. and Yang, L. (2006). Cellular, molecular, and matrix changes in cartilage during aging and osteoarthritis. J. Musculoskelet. Neuronal Interact. 6, 379381.

Joeng, K. S., Song, E. J., Lee, K. J. and Lee, J. (2004). Long lifespan in worms with long telomeric DNA. Nature Genetics 36, 607-611.

Joyner, M. J. (2008). Viewpoint: not so fast: Intrinsic heart rate vs. $\beta$-adrenergic responsiveness in the aging human heart. J. Appl. Physiol. 105, 3-4.

Kawahara, T. L. A., Michishita, E., Adler, A. S., Damian, M., Berber, E., Lin, M., McCord, R. A., Ongaigui, K. C. L., Boxer, L. D., Chang, H. Y. and Chua, K. F. (2008). SIRT6 links histone $\mathrm{H} 3$ lysine 9 deacetylation to NF-kB-dependent gene expression and organismal life span. Cell 136, 62-74.

Kim, S. K. (2007). Common aging pathways in worms, flies, mice and humans. J. Exper. Biol. 210, 1607-1612.

Kirkwood, T. B. L. (2005). Understanding the odd science of aging. Cell 120, 437-447.

Kirkwood, T. B. L. (2008). A systematic look at an old problem. Nature 451, 644-647.

Kirkwood, T. B. L. and Holliday, R. (1979). The evolution of ageing and longevity. Proc. R. Soc. Lond. B Biol. Sci. 205, 531-546.

Kriete, A. and Mayo, K. L. (2009). Atypical pathways of NFkappa B activation and aging. Exp. Gerontol. 44, 250-255.

Lee, J. H., O'Keefe, J. H., Bell, D., Hensrud, D. D. and Holick, M. F. (2008). Vitamin D deficiency: An important, common, and easily treatable cardiovascular risk factor? J. Am. Coll. Cardiol. 52, 1949-1956.

Lips, P. (2006). Vitamin D physiology. Prog. Biophys. Mol. Biol. 92, 4-8.

Ljubuncic, P. and Reznick, A. Z. (2009). The evolutionary theories of aging revisited - a mini-review. Gerontol. 55, 205-216.

Llewellyn, D. J., Langa, K. and Lang, I. (2009). Serum 25hydroxyvitamin $\mathrm{D}$ concentration and cognitive impairment. $\mathrm{J}$. Geriatr. Psychiatry. Neurol. (online)

Medawar, P. B. (1952). An Unsolved Problem of Biology. London, H.K. Lewis

Mele, J., Van Remmen, H., Vijg, J. and Richardson, A. (2006). Characterization of transgenic mice that overexpress both copper zinc superoxide dismutase and catalase. Antioxid. Redox. Signal. 8, 628-638.

Monnet-Tschud, F., Zurich, M. G., Boschat, C., Corbaz, A. and Honegger, P. (2006). Involvement of environmental mercury and lead in the etiology of neurodegenerative diseases. Rev. Environ. Health 21, 105-117.

North, B. and Verdin, E. (2004). Sirtuins: Sir2-related NADdependent protein deacetylases. Genome Biol. 5, 224-235.

Ornish, D., Lin, J., Daubenmier, J., Weidner, G., Epel, E., Kemp,
C., Jesus, M., Magbanua, M., Marlin, R., Yglecias, L., Carroll, P. R. and Blackburn, E. H. (2008). Increased telomerase activity and comprehensive lifestyle changes: a pilot study. Lancet 9, 1048-1057.

Pamplona, R. (2008). Membrane phospholipids, lipo-oxidative damage and molecular integrity: a causal role in aging and longevity. Biochim. Biophys. Acta 1777, 1249-1262.

Parrinello, S., Coppe, J. P., Krtolica, A. and Campisi, J. (2005). Stromal-epithelial interactions in aging and cancer: senescent fibroblasts can alter epithelial cell differentiation. J. Cell Sci. 118, 485-496.

Pérez, V. I., Van Remmen, H., Bokov, A., Epstein, C. J., Vijg, J. and Richardson, A. (2009). The over-expression of major antioxidant enzymes does not extend the lifespan of mice. Aging Cell 8, 73-75.

Pérez-Rivero, G., Ruiz-Torres, M. P., Díez-Marqués, M. L., Canela, A., López-Novoa, J. M., Rodríguez-Puyol, M., Blasco, M. A. and Rodríguez-Puyol, D. (2008). Telomerase deficiency promotes oxidative stress by reducing catalase activity. Free Radic. Biol. Med. 45, 1243-1251.

Richards, J. B., Valdes, A. M., Gardner, J. P., Paximadas, D., Kimura, M., Nessa, A., Lu, X., Surdulescu, G. L., Swaminathan, R., Spector, T. D. and Aviv, A. (2007). Higher serum vitamin $D$ concentrations are associated with longer leukocyte telomere length in women. Am. J. Clin. Nutr. 86, 1420-1425.

Sansoni, P., Vescovini, R., Fagnoni, F., Biasini C., Zanni, F., Zanlari, L., Telera, A., Lucchini, G., Passeri, G., Monti, D., Franceschi, C. and Passeri, M. (2008). The immune system in extreme longevity. Exp. Gerontol. 43, 61-65.

Schriner, S. E., Linford, N. J., Martin, G. M., Treuting, P., Ogburn, C. E., Emond, M., Coskun, P. E., Ladiges, W., Wolf, N., Van Remmen, H., Wallace, D. C. and Rabinovitch, P. S. (2005). Extension of murine life span by overexpression of catalase targeted to mitochondria. Science 308, 1909-1911.

Sinclair, D. A. (2005). Toward a unified theory of caloric restriction and longevity regulation. Mech. Ageing Dev. 126, 987-1002.

Steinbrenner, H. and Sies H. (2009). Protection against reactive oxygen species by selenoproteins. Biochim. Biophys. Acta. Mar 5 (In press).

Varady, K. A., Roohk, D. J., McEvoy-Hein, B. K., Gaylinn, B. D., Thorner, M. O. and Hellerstein, M. K. (2008). Modified alternate-day fasting regimens reduce cell proliferation rates to a similar extent as daily calorie restriction in mice. FASEB J. 22, 2090-2096.

Vijg, J., Maslov, A.Y. and Suh, Y. (2008). Aging: a sirtuins shake-up? Cell 135, 797-798.

Warren, L. A. and Rossi, D. J. (2009). Stem cells and aging in the hematopoietic system. Mech. Aging Dev. 130, 46-53.

Williams, G. C. (1957). Pleiotropy, natural selection, and the evolution of senescence. Evolution 11, 398-411.

Witte, A. V., Fobker, M., Gellner, R., Knect, S. and Flöel, A. (2009). Caloric restriction improves memory in elderly humans. Proc. Natl. Acad. Sci. USA. 106, 1255-1260. 